07;08

\title{
Потребление энергии для высокочастотного переключения микродискового лазера с квантовыми точками
}

\author{
(C) А.Е. Жуков ${ }^{1,2}$, Э.И. Моисеев ${ }^{1}$, Н.В. Крыжановская ${ }^{1}$, Ф.И. Зубов ${ }^{1}$, А.М. Можаров ${ }^{1}$, Н.А. Калюжный ${ }^{3}$, \\ С.А. Минтаиров ${ }^{3}$, М.М. Кулагина ${ }^{3}$, С.А. Блохин ${ }^{3}$, М.В. Максимов ${ }^{1,3}$ \\ ${ }^{1}$ Санкт-Петербургский национальный исследовательский Академический университет РАН, Санкт-Петербург, Россия \\ ${ }^{2}$ Санкт-Петербургский политехнический университет Петра Великого, Санкт-Петербург, Россия \\ ${ }^{3}$ Физико-технический институт им. А.Ф. Иофрфе РАН, Санкт-Петербург, Россия \\ E-mail: zhukale@gmail.com
}

Поступило в Редакцию 22 мая 2019 г.

В окончательной редакции 22 мая 2019 г.

Принято к публикации 23 мая 2019 г.

Исследованы характеристики микродискового лазера диаметром $23 \mu \mathrm{m}$ в режиме прямой высокочастотной модуляции при стабилизированной температуре теплоотвода $18^{\circ} \mathrm{C}$. Показано, что минимальное потребление электроэнергии составляет $\sim 1.6 \mathrm{pJ} / \mathrm{bit}$ и достигается при частоте модуляции $4.2 \mathrm{GHz}$. Максимальная частота модуляции составляет $6.7 \mathrm{GHz}$, при этом энергопотребление равно $3.3 \mathrm{pJ} / \mathrm{bit}$.

Ключевые слова: высокочастотная модуляция, микролазер, полупроводниковый лазер, квантовые точки, энергопотребление.

DOI: 10.21883/PJTF.2019.16.48158.17885

Полупроводниковые микродисковые/микрокольцевые лазеры $[1,2]$ представляют интерес для различных приложений, требующих источников оптического излучения, обладающих компактными размерами. При диаметре $10-30 \mu \mathrm{m}$ микродисковые лазеры с активной областью на основе квантовых точек способны работать в непрерывном режиме, в том числе без термостабилизации, вплоть до $110^{\circ} \mathrm{C}[3]$, пороговые токи при комнатной температуре составляют единицы $\mathrm{mA}$. В отличие от поверхностно-излучающих лазеров с вертикальным микрорезонатором микродисковые лазеры с квантовыми точками могут быть изготовлены на основе относительно простых, не содержащих многослойных отражателей эпитаксиальных гетероструктур, в том числе синтезированных на инородных подложках (кремнии $[4,5]$ ), а для изготовления резонатора может быть использована фотолитография с последующим травлением глубокой мезы без необходимости пассивации боковых граней.

Наиболее перспективно применение таких микролазеров для реализации систем оптической связи на кристалле. Недавно нами была продемонстрирована возможность достижения прямой модуляции в гигагерцевом диапазоне частот [6] с помощью микродисков, содержащих плотные массивы квантовых островков InGaAs (так называемые квантовые ямы-точки) [7]. При работе без термостабилизации максимальная частота модуляции составила $5.9 \mathrm{GHz}$. В настоящей работе нами было предпринято исследование высокочастотных характеристик микродискового лазера, работающего в режиме стабилизации температуры теплоотвода. Также нами исследованы электрические потери, возникающие при переключении микролазера. Показано, что при размерах лазерного излучателя около $20 \mu \mathrm{m}$ электрические потери могут быть менее $2 \mathrm{pJ} / \mathrm{bit}$, что существенно ниже ранее сообщенных значений для микролазеров на подложках InP сравнимых размеров, для которых наименьшее значение составило около $7 \mathrm{pJ} / \mathrm{bit}$ [8].

Эпитаксиальная структура была синтезирована на подложке GaAs n-типа проводимости методом газофазной эпитаксии из металлоорганических соединений и представляла собой лазерную гетероструктуру $\mathrm{AlGaAs} / \mathrm{GaAs}$ с раздельным ограничением носителей заряда и оптической моды. В активной области было сформировано пять рядов квантовых ям-точек InGaAs. Детальное описание послойной конструкции приведено в [3]. Микродисковые

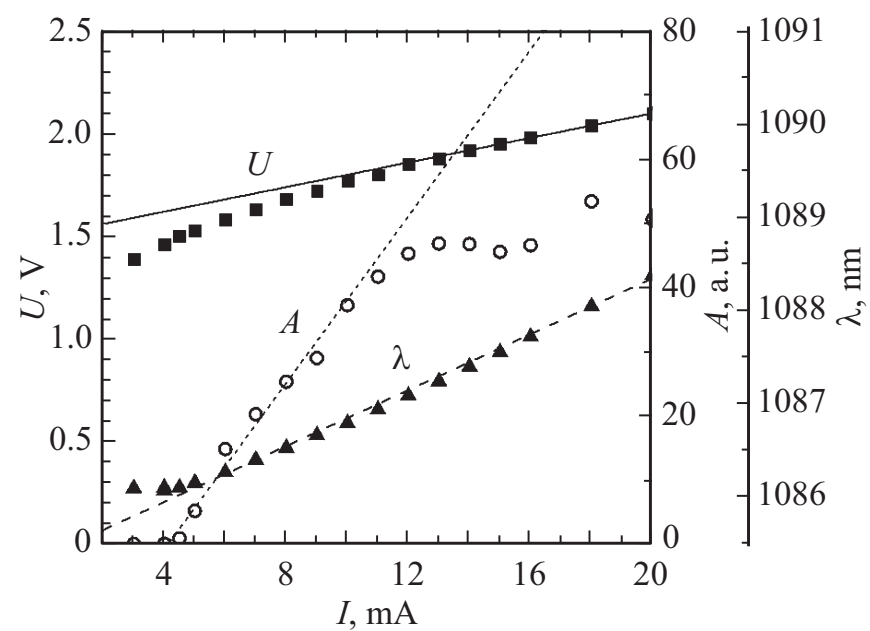

Рис. 1. Зависимость от тока смещения $I$ падения напряжения $U$ (квадраты), интегральной интенсивности лазерной линии $A$ (кружки) и ее спектрального положения $\lambda$ (треугольники). Прямые - линейная аппроксимация. 


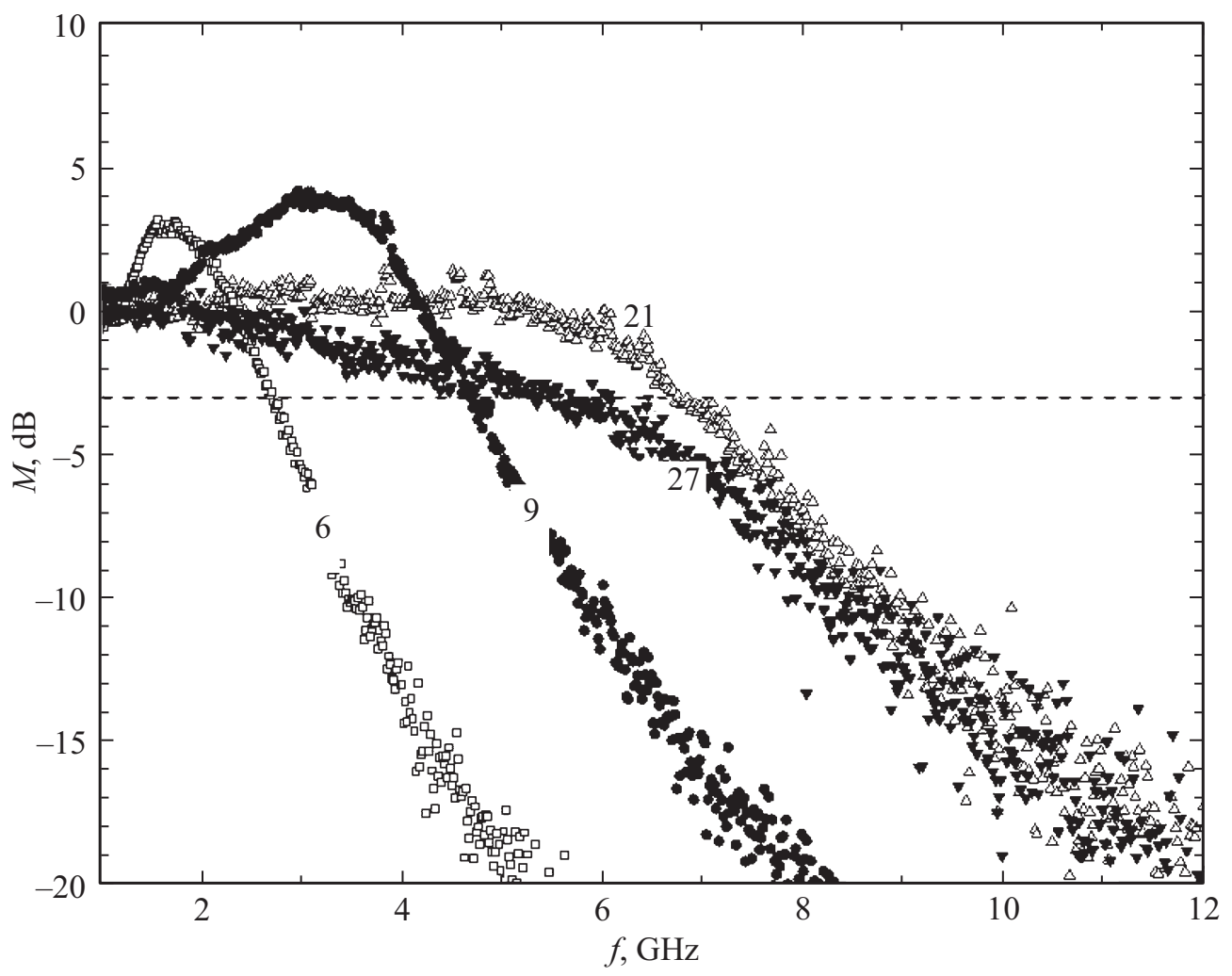

Рис. 2. Амплитудно-частотные характеристики, записанные при различном токе смещения. Числа около кривых соответствуют величине тока (в $\mathrm{mA})$, штриховая линия - уровню $-3 \mathrm{~dB}$.

лазеры были изготовлены с помощью оптической фотолитографии и последующего глубокого плазмохимического травления и затем планаризованы резистом SU-8. Микролазеры были напаяны общим n-контактом на теплоотвод. Индивидуальное электрическое соединение к контакту $p$-типа осуществлялось с помощью золотой проволоки.

Для исследований был отобран микродисковый лазер диаметром $23 \mu \mathrm{m}$. Измерения проводились при фиксированной температуре теплоотвода, которая была установлена равной $18^{\circ} \mathrm{C}$. Вольт-амперная характеристика и порог генерации определялись в непрерывном режиме. Для высокочастотных измерений использовался зонд типа ground-signal-ground (GSG). Измерения проводились при прямом смещении постоянным током с одновременной гармонической модуляцией током малой амплитуды (малосигнальная прямая модуляция). Частота модуляции варьировалась в пределах $0.1-20 \mathrm{GHz}$, ток смещения до $30 \mathrm{~mA}$.

На рис. 1 показана вольт-амперная характеристика микролазера, имеющая свойственный диодам вид. В области больших токов инжекции прибор характеризуется дифференциальным сопротивлением $30 \Omega$ (сплошная линия). На рисунке также приведена зависимость интегральной интенсивности линии излучения от тока накачки, которая позволяет оценить порог генерации как $4.2 \mathrm{~mA}$ по началу быстрого увеличения интенсивности линии (пунктирная линия). С увеличением тока положение линии генерации сдвигается в длинноволновую сторону со скоростью около $0.15 \mathrm{~nm} / \mathrm{mA}$ (штриховая линия). Учитывая, что температурный сдвиг моды шепчущей галереи в таких микрорезонаторах составляет около $0.08 \mathrm{~nm} / \mathrm{K}$ [9], можно оценить перегрев активной области относительно фиксированной температуры теплоотвода, составляющий около $2^{\circ} \mathrm{C} / \mathrm{mA}$.

На рис. 2 показана эффективность амплитудной модуляции $M$ в зависимости от частоты $f$ изменения модулирующего тока (амплитудно-частотная характеристика, АЧХ), измеренная при нескольких токах смещения I. Мерой полосы модуляции лазерного излучения при данном токе смещения полагают частоту $f_{3 \mathrm{~dB}}$, которая соответствует уменьшению эффективности модуляции в 2 раза (до уровня $-3 \mathrm{~dB}$ ) относительно ее низкочастотного значения, для которого $M$ принимают равным единице. Как видно, при малых токах смещения АЧХ демонстрирует выраженный резонансный пик, обусловленный релаксационными колебаниями [10]. При увеличении тока свыше порога генерации частота $f_{3 \mathrm{~dB}}$ быстро возрастает. При дальнейшем росте тока резонансный пик исчезает (говорят, что релаксационные колебания демпфированы), а величина $f_{3 \mathrm{~dB}}$ достигает своего предельного значения, после чего снижается. Для исследованного микролазера максимальная величина $f_{3 \mathrm{~dB}}$ составила $6.7 \mathrm{GHz}$, что, насколько нам известно, 


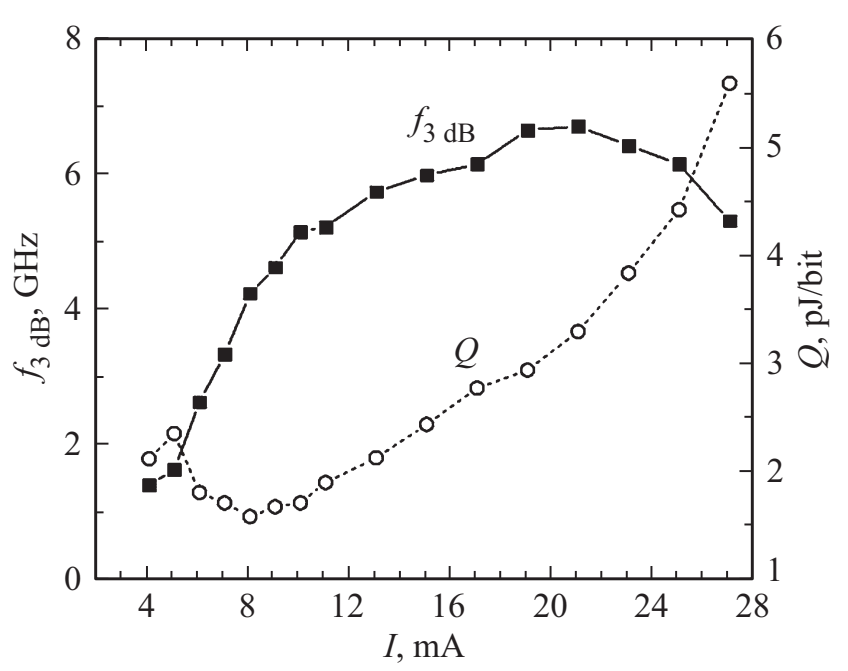

Рис. 3. Зависимость определенной по уровню $-3 \mathrm{~dB}$ максимальной частоты модуляции $f_{3 \mathrm{~dB}}$ (квадраты) и величины электрических потерь на переключение $Q$ (кружки) от тока смещения.

является наибольшей полосой модуляции, измеренной для микролазеров сравнимого размера на основе квантовых точек.

Энергия $Q$, потребляемая лазером для передачи одного бита информации (energy-to-data ratio, EDR), может быть оценена как отношение электрической мощности к удвоенной полосе модуляции [11]:

$$
Q=\frac{U I}{2 f_{3 \mathrm{~dB}}} .
$$

Рис. 3 обобщает зависимость $f_{3 \mathrm{~dB}}$ и $Q$ от тока смещения микролазера. Вблизи порога генерации $I_{t h}$ величина электрических потерь снижается с ростом тока, что обусловлено быстрым увеличением $f_{3 \mathrm{~dB}} \propto \sqrt{I-I_{t h}}$. Наименьшее значение $Q$ достигается при токе смещения $8 \mathrm{~mA}$, приблизительно в 2 раза превосходящем $I_{t h}$, и составляет $1.58 \mathrm{pJ} / \mathrm{bit}$. Соответствующая частота $f_{3 \mathrm{~dB}}$ превышает $4 \mathrm{GHz}$. Дальнейшее увеличение смещения ведет к возрастанию $Q$, вызванному как замедлением роста $f_{3 \mathrm{~dB}}$, так и увеличением электрической мощности, в которой начинает заметную роль играть тепловая мощность, рассеиваемая на последовательном сопротивлении лазера. Току смещения, отвечающему наибольшему быстродействию микролазера, соответствует величина $Q=3.3 \mathrm{pJ} / \mathrm{bit}$.

Таким образом, нами были исследованы электрические потери и быстродействие микродискового лазеpa c квантовыми ямами-точками в активной области в режиме прямой модуляции с температурностабилизированным теплоотводом. Достигнуто существенное (примерно в 2 раза) уменьшение потребления энергии в расчете на 1 bit по сравнению с ранее исследованными нами неохлаждаемыми микролазерами аналогичной конструкции [12].

\section{Финансирование работы}

Работа выполнена при поддержке Российского научного фонда (соглашение 19-72-30010).

\section{Конфликт интересов}

Авторы заявляют, что у них нет конфликта интересов.

\section{Список литературы}

[1] McCall S.L., Levi A.F.J., Slusher R.E., Pearton S.J., Logan R.A. // Appl. Phys. Lett. 1992. V. 60. N 3. P. 289-291.

[2] Mao M.-H., Chien H.-C., Hong J.-Z., Cheng C.-Y. // Opt. Express. 2011. V. 19. N 15. P. 14145-14151.

[3] Moiseev E., Kryzhanovskaya N., Maximov M., Zubov F., Nadtochiy A., Kulagina M., Zadiranov Yu., Kalyuzhnyy N., Mintairov S., Zhukov A. // Opt. Lett. 2018. V. 43. N 19. P. 4554-4557.

[4] Kryzhanovskaya N., Moiseev E., Polubavkina Yu., Maximov M., Kulagina M., Troshkov S., Zadiranov Yu., Guseva Yu., Lipovskii A., Tang M., Liao M., Wu J., Chen S., Liu H., Zhukov A. // Opt. Lett. 2017. V. 42. N 17. P. 3319-3322.

[5] Wan Y., Norman J., Li Q., Kennedy M.J., Liang D., Zhang C., Huang D., Zhang Z., Liu A.Y., Torres A., Jung D., Gossard A.C., Hu E.L., Lau K.M., Bowers J.E. // Optica. 2018. V. 4. N 8. P. $940-944$.

[6] Kryzhanovskaya N.V., Moiseev E.I., Zubov F.I., Mozharov A.M., Maximov M.V., Kalyuzhnyy N.A., Mintairov S.A., Kulagina M.M., Blokhin S.A., Kudryavtsev K.E., Yablonskiy A.N., Morozov S.V., Berdnikov Yu., Rouvimov S., Zhukov A.E. // Photon. Res. 2019. V. 7. In press.

[7] Nadtochiy A.M., Maximov M.V., Mintairov S.A., Kalyuzhnyy N.A., Rouvimov S., Zhukov A.E. // J. Phys.: Conf. Ser. 2017. V. 917 . P. 032001 (1-4).

[8] Yang Y.-D., Zhang Y., Huang Y.-Z., Poon A.W. // Opt. Express. 2014. V. 22. N 1. P. 824-838.

[9] Kryzhanovskaya N.V., Moiseev E.I., Kudashova Yu.V., Zubov F.I., Lipovskii A.A., Kulagina M.M., Troshkov S.I., Zadiranov Yu.M., Livshits D.A., Maximov M.V., Zhukov A.E. // Electron. Lett. 2015. V. 51. N 17. P. 1354-1355.

[10] Semiconductor lasers I. Fundamentals / Ed. E. Kapon. San Diego, CA, USA: Academic Press, 1999. P. 303-305.

[11] Moser P., Hofmann W., Wolf P., Lott J.A., Larisch G., Payusov A., Ledentsov N.N., Bimberg D. // Appl. Phys. Lett. 2011. V. 98. N 23. P. 231106 (1-3).

[12] Zhukov A.E., Kryzhanovskaya N.V., Maximov M.V. Microdisk lasers: a new opportunity for self-organized quantum dots // Int. Conf. „Frontiers of 21st Century Physics and Ioffe Institute“. Abstract book. St. Petersburg, 2018. P. 33-34. 